\title{
THE APPLICATION OF EXPANDABLE ENDOPROSTHETIC RECONSTRUCTION FOR LIMB SALVAGE SURGERY IN THE SKELETALLY IMMATURE PATIENTS
}

\author{
RONG-SEN YANG \\ Department of Orthopaedics, College of Medicine, National Taiwan University, Taipei, Taiwan
}

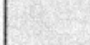

ABSTRACT

The development of new image techniques enables the early detection of bone tumors in the extremities in the past two decades. In addition, new advance of adjuvant therapy improves the longterm survival of patients with primary bone malignancies. Since the primary bone malignancy frequently occurs at the metaphysis of long bones of pediatric patients in the first and second decade, many patients have to face the late problems of limb length discrepancy after the limb salvage operation. Since the inevitable limb length discrepancy will interfere the functional outcomes and cosmetic appearance, the reconstruction of limb defect after limb salvage remains a challenge. Several options of reconstruction are available for these patients, including resection arthrodesis, rotationplasty, osteochondral allograft reconstruction, and endoprosthetic reconstruction. All these methods are difficult to address the limb length discrepancy. The development of expandable endoprosthetic reconstruction makes limb-salvage surgery feasible in the skeletally immature and provides another choice of solution. This article presents the current status of custom-expandable endoprosthetic reconstruction in the skeletally immature patients after wide resection of primary bone malignancies in the extremity. The surgical options, complications and functional results will be emphasized.

Basically these expandable endoprostheses can be classified according to the expansion design. Recent reports demonstrated that the results of expandable prosthesis in the growing children are rather acceptable. Some patients can regain the equal limb length after expansion of the prosthesis. However these patients have to take several expansion procedures for the equality of limb length during the growing period. A rather high complication rate of either endoprosthesis-related or diseaseassociated still needs to be settled in the near future. These include mechanical failure of the expansion mechanism, extensive metallosis, aseptic loosening, fatigue fracture, flexion contracture, local recurrence, delayed wound healing, fat embolism, local overgrowth of counterpart bone, nerve palsy, infection, and bone fracture. Some patients even require an amputation even after expandable endoprosthesis reconstruction because of difficult reconstruction or severe functional impairment. However, with regard to difficult rehabilitation for patients under 8 years, amputation or alternative options need to be considered. A comprehensive discussion with the parents and patient about the detailed treatment protocol is needed before performing reconstruction using expandable endoprosthesis.
\end{abstract}

Biomed Eng Appl Basis Comm, 2001 (June); 13: 141-147.

Received: May 25, 2001; accepted: June 10, 2001 Correspondence: Rong-Sen Yang, Professor, Department of Orthopaedics,

National Taiwan University Hospital

No. 7, Chung-Shan South Road, Taipei, TAIWAN

E-mail: Yang@ha.mc.ntu.edu.tw
INTRODUCTION-With Special Emphasis on the Limb Length 
The current treatment options for patients with primary bone malignancies emphasize the best realistic survival rate and local control of tumor, as well as the best possible functional outcomes and psychological acceptance [1-5]. New era of current adjuvant chemotherapy in the recent three decades enables the limb salvage for the properly selected patients with primary bone malignancies in the extremity. The current studies have proven it to be equivalent in both survival and local control of tumor as compared to traditional amputation [4-7].

The primary bone malignancy usually affects the metaphysis of long bones of young patients in the first and second decade. Therefore these skeletally immature patients usually have a growing growth plate involved. Since the basic goal of limb salvage is to widely resect the local tumor and to preserve a wellfunctioning, tumor-free, and painless limb that can improve body balance, simplify the rehabilitation process and preserve intact body image [8]. Therefore a successful limb salvage operation usually needs to widely excise the tumor with a safe margin, i.e., usually including a growth plate near the metaphysis. This will impair the continuous growth of limb length in the long-term survival period. On the other hand, the normal contra-lateral limb with normal growth plate will continue its growth and result in marked limb length inequality. Such a problem renders the reconstruction of a large segmental defect in skeletally immature a difficult challenge [1-20].

\section{Operation Options for the Skeletally Immature Patients with Bone Malignancy}

The conventionally recommended treatment for these young pediatric patients is amputation. The amputation ensures the radical excision of bone tumor and all patients need to put on an exoprosthesis for their activity. They have to take part in an active rehabilitation program that may seem difficult for them. In addition, they have to change their prosthesis when the leg length discrepancy occurs. Pediatric patients having high proximal amputees with external prostheses need even more difficult gait training and frequent prosthetic replacement as they grow $[5,6,21]$.

Recently the requirement of a better limb function and intact body image, as previously described, have stimulated the development of limb salvage protocol that means an adequately wide resection of tumor followed by reconstruction of the limb. Before the advent of expandable endoprosthesis, the available surgical options for reconstruction after limb salvage in the skeletally immature include resection arthrodesis with a bone graft (vascularized or non-vascularized, an autograft or allograft) or bone cement, rotationplasty, osteochondral allograft reconstruction, and conventional endoprosthetic reconstruction $[3,4,6,12,22-24]$. The resection arthrodesis can provide a stable limb, but at the expense of limited motion and potential risk of fracture or infection. The resection of bone tumor followed by osteochondral allograft reconstruction can also provide a joint with rather acceptable function. However, it has a potential risk of transmission of disease, arthritis, fracture, and growth of the allograft is questionable. The rotationplasty can provide a biological reconstruction, but with the drawback of bizarre appearance and need of external prosthesis [3]. On the other hand, small bone size and non-growing potential of conventional endoprosthesis preclude its implantation in skeletally immature [7]. All these reconstruction options cannot address the problems of eventually limb length inequality that is less cosmetically acceptable and reduces the functional quality $[4,5,7,12,20,21]$. Although unequal limb length can be managed by the epiphysiodesis of the normal limb, it may result in varus or valgus deformity, inappropriate proportion of limb segment, and inevitable loss of height. All these procedures may cause potential cosmetic or psychological problems $[1,2,6,9,10,25]$.

Since the resection of a major growth plate of affected limb in the skeletally immature would cause progressive limb length inequality, surgical reconstruction after limb salvage is more difficult than that of the adults $[11,16,18]$. However, the aforementioned treatment options cannot solve the problem of limb length discrepancy when the patients reach skeletal maturity. Therefore, the endoprosthetic reconstruction with expandable prosthesis may provide another option for the skeletally immature with primary bone malignancies.

\section{Development and Designs of Expandable Endoprosthesis}

In 1976, Scales et al. introduced a new concept of expansion and designed the first expandable endoprosthesis [20]. Their preliminary clinical results are promising. The basic goal of the expandable endoprosthetic reconstruction is to overcome the anticipated limb length discrepancy in children with primary bone malignancies involving a major growth plate. In addition, it can provide a painless, functional, length-equal, tumor-free limb $[1,2,6,9,10]$. The initial expandable endoprosthesis also can be revised to adult type prosthesis at the time of skeletal maturity and provide a potentially durable biological fixation $[5,26]$. Expandable endoprosthetic reconstruction can improve function level of limbs without need of external prosthesis, but at the cost of multiple operation procedure. In recent decade, a variety of expandable endoprostheses provide more treatment alternatives for the skeletally immature with primary bone malignancies [4-7,11$17,20,21,25-37]$.

The design and principle of the expansion endoprosthesis has been described previously $[1,2,4-$ $7,9,10,12,17,20,25,30,33,38,39]$. The customexpandable endoprostheses used in the initial operation 
include Dow Corning Wright (DCW) LEAP prostheses (Lewis Expandable Adjustable Prosthesis, Dow Corning Wright, Arlington, Tennessee), Howmedica (HM) modular prostheses (Rutherford, New Jersey), Wright Medical (WM) modular prostheses, Techmedica (TM) prosthesis (Camarillo, California). Recently Howmedica adult-type modular prostheses can be used for these purposes. We have also designed our prostheis with special modular design (Union Prostheisis, Taiwan) that can be used for reconstruction after limb salvage (Fig. 1).

In general, the design of the expandable endoprosthesis can be classified into three types, i.e., endoprosthesis incorporating a telescoping expansion mechanism, conventional modular type endoprosthesis, and endoprosthesis incorporating a self-regulating elongation mechanism $[1,2,4-7,9,10,12,17,20,25,28$, $30,32,33,35-40]$. The basic design of the expansion mechanism of LEAP is a central threaded stem that can be lengthened by inserting a chuck key and rotating it. A weight-sharing sleeve can be inserted into the lengthened gap to protect against the thread collapse $[1,2,6,9,10]$. The size and length of expandable endoprosthesis is dependent on the individual basis. The maximal expansion of each prosthesis is determined by the length of the hollow tube that houses the telescoping shaft. The longer the hollow tube and the telescoping shaft can be, the more expansion length is possible. However, the longer expansion will compromise the strength of prosthesis. Therefore, many prosthesis-related complications may occur. Frequent expansion mechanism failure with the LEAP prostheses, as well as its associated titanium debris, limits its use in special instances. Currently, the smaller prostheses would be replaced with a new expandable prosthesis to get additional expandable capability and strength during the treatment period.

The development of modular system can provide superior flexibility of length during the reconstruction $[1,2,6,9,10]$. The intercalated segment of the modular

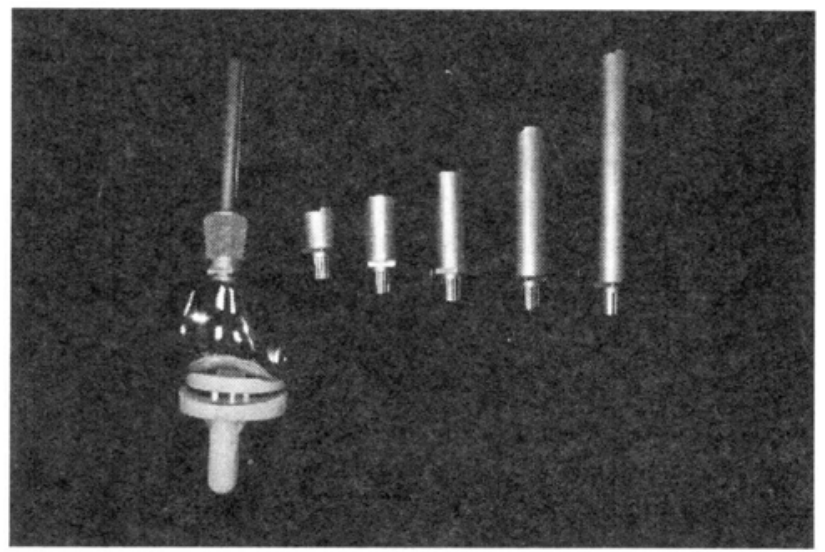

Fig. 1: The modular system of custom-made Union knee prosthesis with segment of various length. prosthesis can be changed with a longer segment when the limb length discrepancy developed later. It can maintain the proper diameter of segment. Therefore such prosthesis can provide more strength than the conventional expandable prosthesis and reduce the risk of fatigue fracture. Modular endoprostheses also provide easier technique for reconstruction or revision to final definite prosthesis. Simpler modular endoprosthetic systems are now being utilized with early good success. Their clinical outcomes are promising. However, the modular prosthesis still needs an invasive approach for expansion. The long-term results need further investigation $[1,2,6,9,10]$.

The conventional expandable endoprostheses are lengthened invasively through multiple operations that would increase the risk of infection and eventually leads to amputation. The new generation of growing endoprosthesis incorporating self-regulating elongation mechanism that can be lengthened by noninvasive methods [37-39]. Such a growing prosthesis can be lengthened by the adjustment of the external magnetic field $[32,35,36,38,40]$ or by knee bending kinetic energy $[37,39]$. Using these types of the endoprostheses, the periprosthetic soft tissue can be lengthened gradually by the traction and decrease the stiffness caused by periprosthetic pseudocapsule in the conventional expandable prostheses $[3,35,37,39]$. These experimental surgery using such types of prostheses is promising, however, both growing endoprostheses are in the initial development stages. Further investigation is necessary for their final clinical significance using this kind of endoprosthesis.

\section{Pre-OP Assessment and Indications for the Expandable Prosthesis}

These expandable endoprosthesis is mainly used for the reconstruction after limb salvage operation in the skeletally immature patients with primary malignant bone tumors of the extremity. The ultimate goal of expandable prostheses is to equalize extremity length at skeletal maturity. Over-expansion or underexpansion will result in poor results. In general, patients with age under 13 years usually need expansion of the prostheses [6,13], while the amount of lengthening should be determined on individual basis. The indication for expandable prosthesis is reserved for those with the potential length discrepancy of 4 to $5 \mathrm{~cm}$ or more. The expected growth less than 2 to $3 \mathrm{~cm}$ can be over-lengthened at initial operation $[4,25,31]$. However, the prediction of limb length in the skeletally immature is a complicated process that includes an assessment with bone age films and calculation the amount of remaining growth by the graphs of Anderson et al. [4,5,8]. The body heights of parents and siblings are important parameters for estimation. However, the growth chart is different for different races. Furthermore, many parameters can be changed during 
these three decades, including the nutrition, sports activities, etc. In addition, the limb length of upper extremities usually is not studied. Therefore, an update growth chart is necessary for the more exact prediction of ultimate limb length in the growing child.

The reported diagnosis includes mainly the parosteal osteosarcoma, osteosarcoma, Ewing's sarcoma, and some other malignant tumors. The locations of the tumors included proximal femur, distal femur, proximal humerus, and proximal tibia. All patients attempted a wide resection of tumor and received the adjuvant therapy or neoadjuvant therapy. Before operation, patients and parents must be informed of the need of early mobilization, gait training, multiple expansion procedures, the possibility of replacement of the prosthesis, and eventual conversion to a standard endoprosthesis at skeletal maturity only if clinically indicated-mechanical failure or loosening $[5,6]$. The comprehensive communication and cooperation is essential for a successful reconstruction using expandable endoprosthesis.

\section{Surgical Techniques, Indications for Expan- sion, and Post-Operative Rehabilitation}

The surgical approach for limb salvage surgery and the implantation technique of expandable prostheses are described previously [5]. In brief, a longitudinal incision is made and followed by the excision of the previous biopsy site and the wide dissection of tumor. The osteotomy is usually done at least $5 \mathrm{~cm}$ away from the tumor extent. For the distal femur tumor, the patella and major portion of the quadriceps mechanism are always preserved. Medial gastrocnemius flap is used for reconstruction for proximal tibia tumor. The intramedullary canal is then meticulously prepared and the components are cemented slowly under relative hypotensive anesthesia. In the proximal tibia, distal femoral, and total femur replacements, the patella is also resurfaced using a cemented polyethylene component. During the expansion procedure, periprosthetic pseudocapusle is usually needed to be totally excised (Fig. 2). In order to get patients into early rehabilitation process, it is not necessary to over-lengthen the affected limb at the initial surgery. The postoperative care and rehabilitation program are the same as previously reported [5]

During the postoperative follow-up evaluation, the local recurrence, distant metastasis, infection or prosthetic loosening are evaluated by clinical examination and plain radiograms of the involved limb at each visit, chest CT scans, and technetium-99 bone scans regularly. Limb length equality is assessed by scanograms of both limbs if clinically indicated.

The expansion of the prosthesis is indicated when patients had a limb length discrepancy greater than 1.5 to $2 \mathrm{~cm}$. Some authors recommended a primary lengthening at the initial operation $[4,6,13,14,41]$, whereas others did not advocate it in order to reduce the obstacles in the initial rehabilitation. Many authors lengthened the limb using multiple small lengthening through a small incision over the Jacob's chuck or other gear-expansion mechanism to expand the prosthesis $[4,6,20,21,31,33]$. Each time the expansion length ranged from 1 to $2 \mathrm{~cm}[4-6,13,14,29,41]$. However, the periprosthetic pscudocapsule cannot be excised using this approach. Tightening of this thick pseudocapsule after expansion of 1.5 to $2 \mathrm{~cm}$ would occur and reduce range of motion. Thus Eckardt emphasized the importance of a complete periprosthetic pseudocapsulectomy during expansion [10]. A complete pseudocapsulectomy can distribute the surrounding soft tissue stress, help expansion and improve motion at each procedure. However, careful and skillful dissection of the periprosthetic pseudocapsule is essential to avoid complication since it is close to the neurovascular bundle that is dissected at initial operation.

Although there is a generalized diminished longitudinal growth in all the bones of the affected leg following a growing endoprostheses replacement of the distal femur [42], continuous tibial physeal growth did occur that has been described previously $[5,43]$. Fortunately the preservation of proximal tibial physeal plate after distal or total femoral replacement can contribute some ultimate leg length and delayed or eliminated the indication of expansion. In addition, the upper limb length discrepancy is not so important functionally as compared with the lower extremities, the indication for the expansion of the humerus is less strict than that for the femur or tibia. Patient may tolerate larger limb length discrepancy up to $5 \mathrm{~cm}$ without major functional impairment.

\section{Clinical Outcomes}

Functional outcomes after limb salvage surgery are related to the anatomical location, level of resection and amount of muscle loss, nature and amount of any preoperative adjuvant treatment, tumor size and grade, resection technique, stability of the reconstruction, type and intensity of postoperative rehabilitation, and the patient's cooperation and motivation $[5,26]$. The preservation of an extremity aids in activities of daily living, balance, cosmetics and psychological identity that compared favorably with amputations and external prostheses $[6,19]$. The overall rating of functional outcomes showed that the good to excellent results of expandable endoprosthesis ranged from $38 \%$ to $75 \%[4,10,14,29,31]$. In general, patients with expandable prosthesis had a good to excellent result at the knee, fair to good at the hip, and fair about the shoulder [10]. Usually, the patients with tibial endoprosthesis tend to have a lower functional score than those with the femoral endoprostheses. This may be related to difficult soft tissue reconstruction due to larger 


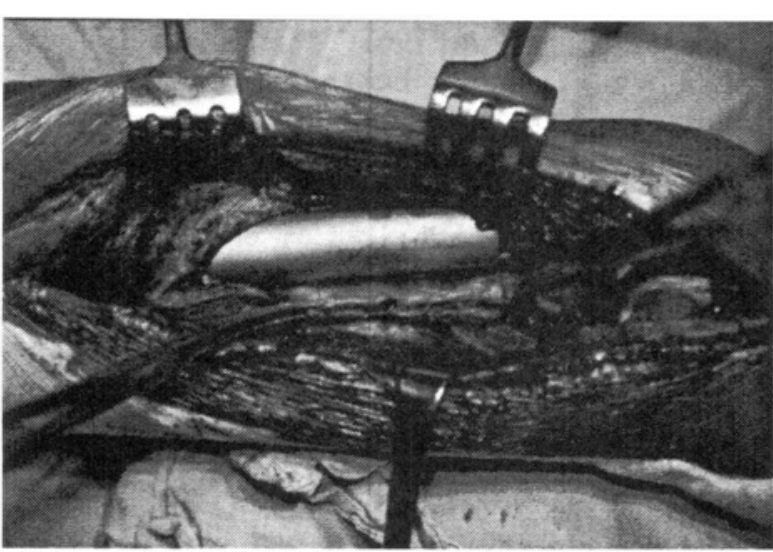

(a)

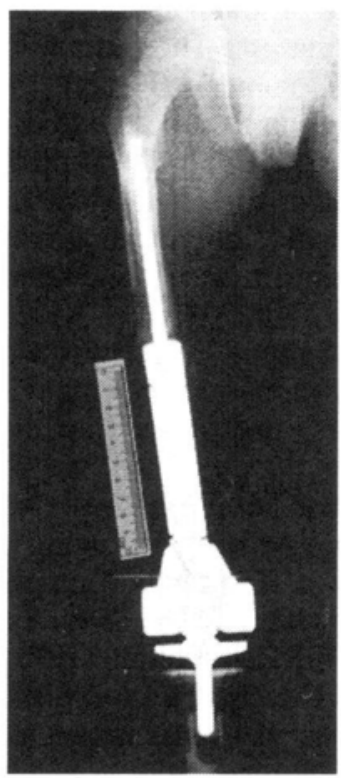

(b)

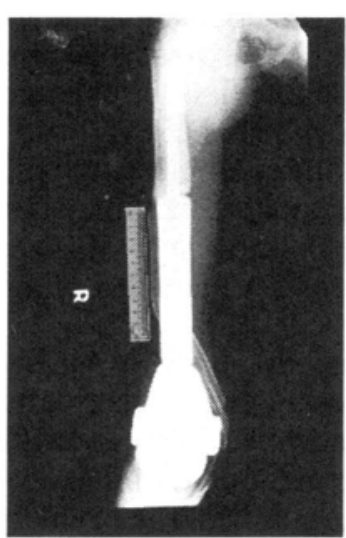

(c)

Fig.2 The periprosthetic pseudocapusle is usually needed to be totally excised during the expansion procedure (A). The pre-lengthening radiograph (B) and post-lengthening radiograph (C) showed an elongation of stem after change with a longer segment. of the limb-salvage cffort by is consistent with findings previously reported in limb salvage surgery $[5,24,26]$.

The long-term durability of expandable endoprostheses remains further study. The continuous remodeling of the medullary cavity and shape in growing child would affect long-term fixation of endoprosthesis [14]. Although published laboratory biomechanical studies of expandable endoprostheses disclosed no signs of thread deformation, wear, seizing, or galling under vigorous cyclic loading testing [33], in vivo series did show thread deformation and expansion mechanism failure ranging from 4 to $54 \%[4,5,31,41,44]$.

The reported revision rate of expandable endoprosthesis ranged from $8.5 \%$ to $38.3 \%$ and increased to $85.7 \%$ after 5 years $[26,29]$.

\section{Complications of the Expandable Endopros- thesis}

The high rate of complications after using expandable endoprosthesis remains a challenge in the orthopaedic oncological practice. The complications after implantation of expandable endoprostheses can be categorized into early and late complication. Early complications included infection (range, $0 \%$ to $38 \%$ ) $[5,15,25,26,31,41,44]$, nerve palsy (range, $3.9 \%$ to $8.3 \%)[5,7,31]$, fat embolism, and delayed wound healing. Late complications included bone fracture (range, $1.3 \%$ to $10.5 \%$ ) [7,31,41], fatigue fracture $(1.7 \%)$ [26], transient circulatory disturbance (range, 5-8\%) [7,41], collapse of expansion mechanism $[9,10]$, local recurrence (range, $4 \%$ to $11 \%$ ) $[3,4,14,26,29-31,44]$, metallosis $[9,10]$, overgrowth of counterpart bone, and aseptic loosening (range, $7.9 \%$ to $47 \%$ ) [5,10,13$15,31,41]$. Some patients may even need an amputation finally because of difficulty of reconstruction, marked contracture, or severc functional ompairment.

The limited range of motion in the adjacent joints is frequent after expansion of prosthesis $[9,10]$. Flexion contracture developed especially in the knee of patients with age under 10 years. The previous studies reported that flexion contracture rate ranged from $9 \%$ to $33 \%[26,31,41]$. The main underlying cause of flexion contracture related to periprosthetic pseudocapsule formation after the step-by-step elongation of the prosthesis of more than $1.5 \mathrm{~cm}[5,26,31,32,37,39]$. Total excision of the periprosthetic pseudocapsule will help relcase the further contracture after lengthening [5,41]. Intensive motion therapy and non-steroid antiinflammatory agents arc also advocated to prevent development of flexion contracture [41]

\section{CONCLUSION}

In conclusion, although combined therapy improves the survival of pediatric patients with primary 
bone malignancies, surgical treatment of this special patient population remains a challenging task. Wellperformed limb salvage in properly selected patients may provide a functional limb that can improve much the quality of life. Expandable prosthesis is a good option for the reconstruction after wide resection of tumor involving a major growth plate. These prostheses can also act as a temporary spacer until skeletal maturity. However, patients under 8 years usually have significant difficulty with the rehabilitation process and amputation or alternative means of reconstruction need to be considered. Furthermore, the large number of expansion mechanism failure complications with the conventional LEAP prostheses, as well as its associated titanium debris, precludes its use except in special instances. Currently the simpler modular endoprosthetic systems are now being utilized with early good success. However, the limb salvage must be considered on individual bases, including careful radiological and pathological staging evaluation, histologic grade and extent of tumor, the involvement of the surrounding structure, the anatomical location, the patient's age, growth potential and life style. The longterm durability and application of the expandable endoprostheses needs further investigation.

\section{REFERENCES}

1. Yang RS: The rotating-Hinged knee prosthesis for limb salvage reconstruction of the knee. Biomedical Engineering 1999; 11: 289-296.

2. Yang RS: Endoprosthetic reconstruction for limb salvage surgery. Biomed Eng Appl Basis Commun. 1998; 10:23-34.

3. Finn HA, Simon MA: Limb-salvage surgery in the treatment of osteosarcoma in skeletally immature individuals. Clin Orthop 1991; 262:108-118.

4. Kenan S, Lewis MM: Limb salvage in pediatric surgery. The use of the expandable prosthesis. Orthop Clinics N Am 1991, 22:121-131.

5. Eckardt JJ, Safran MR, Eilber FR, Rosen G, Kabo JM: Expandable endoprosthetic reconstruction of the skeletally immature after malignant bone tumor resection. Clin Orthop 1993; 297:188-202.

6. Eckardt JJ, Yang RS, Ward WG, et al. Endoprosthetic reconstruction for malignant bone tumors and nontumorous conditions of bone. In Stauffer RN (Ed):Advances in Operative Orthopaedics, 1995; 3 : 61-83.

7. Kotz R, Schiller C, Windhager R, Ritschl P: Endoprostheses in children- First results. In Langlais $F$, Tomeno B (eds): Limb Slavage. 5th International Symposium, St. Malo, ISOLS-GETO, Berlin, Springer-Verlag, 1991, pp 591-599.

8. Anderson M, Green WT, Messner MB: Growth and predictions of growth in the lower extremity. J
Bone Joint Surg [Am] 1963; 45:1-14.

9. Ward WG, Yang RS, Eckardt JJ: Endoprosthetic bone reconstruction following malignant tumor resection in skeletally immature patients. Orthop Clin N Am 1996; 27:493-502.

10. Eckardt JJ, Kabo JM, Kelly CM, et al. Expandable endoprosthesis reconstruction in skeletally immature patients with tumors. Clin Orthop 2000; 373:51-61.

11. Biehl T, Gradinger R, Thomas W, Hipp E, Aigner R: The new GT custom-made knee joint prosthesis for malignant bone tumors. In Enneking WF (ed): Limb Salvage in Musculoskeletal Oncology. New York, Churchill-Livingstone, 1987, pp 599-604.

12. Eckardt JJ, Eilber FR, Rosen G, Kabo JM, Safran M, Mirra JM: Expandable endoprostheses for the skeletally immature: The initial UCLA experience. In Langlais F, Tomeno B (eds): Limb Slavage. 5th International Symposium, St. Malo, ISOLS-GETO, Berlin, Springer-Verlag, 1991, pp 585-590.

13. Kenan S, Bloom N, Lewis MM: Limb sparing surgery in skeletally immature patients with osteosarcoma: The use of an expandable prosthesis. Clin Orthop 1991; 270:223-230.

14. Kenan S, DeSimone DP, Lewis MM: Limb sparing for skeletally immature patients with osteosarcoma: The expandable prosthesis. Cancer Treat Res 1993; 62:205-211.

15. Kenan S, Lewis MM: Prosthesis longevity in skeletally immature patients following limb sparing surgery. In Brown KLB (ed): Complications of Limb Salvage. 6th International Symposium on Limb Salvage (ISOLS), Montreal, 1991, pp 523-526.

16. Lewis MM: The use of an expandable and adjustable prosthesis in the treatment of childhood malignant bone tumors of the extremity. Cancer 1986; 57:499-502.

17. Lewis MM, Bloom N, Esquieres EM, Kenan S, Ryniker DM: The expandable prosthesis. An alternative to amputation for children with malignant bone tumors. AORN J 1987; 46:457-470.

18. Lewis MM, Spires WP, Bloom N: Extendable prosthesis: An alternative to amputation. In Enneking WF (ed): Limb Salvage in Musculoskeletal Oncology. New York, Churchill-Livingstone, 1987, pp 606-610.

19. Otis JC, Lane JM, Kroll MA: Energy cost during gait in osteosarcoma patients after resection and knee replacement and after above-the-knee amputation. J Bone Joint Surg [Am] 1985; 67:606-611.

20. Scales JT, Sneath RS: The extending prosthesis. In Coombs R, Freidlaender G (eds): Bone Tumour Management. London, Butterworths, 1987, pp 168-177.

21. Lewis MM, Kenan S, Bloom N: The expandable adjustable prosthesis: An alternative to amputation in growing children with malignant tumors of the extremities. In Langlais F, Tomeno B (eds): Limb 
Slavage. 5th International Symposium, St. Malo, ISOLS-GETO, Berlin, Springer-Verlag, 1991, pp 577-584.

22. Cammisa FP, Glasser DB, Otis JC, Kroll MA, Lane JM, Healcy JH: The Van Nes tibia rotationplasty. A functionally viable reconstructive procedure in children who have a tumor of the distal end of the femur. J Bone Joint Surg [Am] 1990; 72:1541

23. Dubousset J: Limb salvage in pediatric patients. In Langlais F, Tomeno B (eds): Limb Salvage. 5th International Symposium, St. Malo, ISOLS-GETO, Berlin, Springer-Verlag, 1991, pp 513-515.

24. Eckardt JJ, Eilber FR, Dorey FJ, Mirra JM: The UCLA experience in limb salvage surgery for malignant tumors. Orthopaedics 1985; 8:612-621.

25. Lewis MM, Pafford J, Spires W: The expandable prosthesis- tumour prostheses for children. In Coombs R, Freidlaender G (eds): Bone Tumour Management. London, Butterworths, 1987, pp 177 183.

26. Kenan S, Lewis MM: Limb sparing surgery in children. The expandable prosthesis. Current trends and controversies after the first ten years. 8th International Symposium on Limb Salvage (ISOLS), Italy, 1995, p 124 .

27. Henssge EJ, Hipp E, Grundei H, Bichl T, Dufek P, Gradinger R: Casted femur-knee endoprosthesis with spongy metal surface and length adaption in situ. In Yamamuro T (ed): New Developments for Limb Salvage in Musculoskeletal Tumors. Tokyo, Springer-Verlag, 1991, pp 643-648.

28. Lewis MM: Current concept: The expandable prosthesis. Bull Hosp Joint Dis Orthop Institute 1985; 45:29-31.

29. May DRW, Walker PS: Design and follow-up of extending prosthetic replacements for children. In Brown KLB (ed): Complications of Limb Salvage. 6th International Symposium on Limb Slavage (ISOLS), Montreal, 1991, pp 505-510.

30. Scales JT, Sneath RS, Wright KWJ: Design and clinical use of extending prostheses. In Enneking WF (ed): Limb Salvage in Musculoskeletal Oncology. New York, Churchill-Livingstone, 1987, pp $52-61$.

31. Sneath RS, Carter SR, Grimer RJ: Growing endoprosthetic replacements for malignant tumours. In Langlais F, Tomeno B (eds): Limb Slavage. 5th International Symposium, St. Malo, ISOLS-GETO, Berlin, Springer-Verlag, 1991, pp 573-578.

32. Soubeiran A: Growing prostheses with noninvasive length control. 8th International Symposium on Limb Salvage (ISOLS), Italy, 1995, p 130.

33. Spires WP, Pafford JJ, Lewis MM: Biomechanical evaluation of an extending adjustable tumor prosthesis in total joint and segmental replacement. In Enneking WF (ed): Limb Salvage in Musculoskeletal Oncology. New York, Churchill-Livingstone,
1987, pp 610-612.

34. Tait RJ, Selmer K, Neff JR: Modular prosthetic systems: An alternative to custom fabricated prostheses. Proceedings 7th International Symposium on Limb Salvage (ISOLS), Singapore, 1993, pp 467-472.

35. Verkerke GJ, Koops HS, Veth RPH, Hom V, Postma A, Grootenboer HJ: First clinical experience with a growing endoprosthesis, A limb saving procedure in children. 8th International Symposium on Limb Salvage (ISOLS), Italy, 1995, p 272.

36. Verkerke GJ, Koops HS, Veth RPH, Oldhoff J, Nielsen HKL, van den Kroonenberg HH, Grootenboer HJ, van Krieken FM: Design of a lengthening element for a modular femur endoprosthetic system. J Eng Med 1989, 203:97-102.

37. Windhager R, Robionek B, Schiller $\mathrm{CH}$, Kotz R: An expandable prosthesis with automatic continuous elongation. Proceedings 7th International Symposium on Limb Salvage (ISOLS), Singapore, 1993, p 379.

38. Verkerke GJ, Koops HS, Veth RPH, Grootenboer HJ, deBoer LJ, Oldhoff J, Postma A: Development and test of an extendable endoprosthesis for bone reconstruction in the leg. Int $\mathrm{J}$ Artificial Organ $1994 ; 17: 155-162$.

39. Windhager R, Kotz R: Non-invasive growing mechanism in total femur replacement. First clinical experiences. 8th International Symposium on Limb Salvage (ISOLS), Italy, 1995, p 125.

40. Verkerke GJ, Koops HS, Veth RPH, van den Kroonenberg HH, Grootenboer HJ, Nielsen HKL, Oldhoff J, Postma A: An extendable modular endoprosthetic system for bone tumour management in the leg. J Biomed Eng 1990; 12:91-96.

41. Windhager R, Schiller CH, Giesinger B, Kotz R: Complications and problems with expandable prostheses in children. Treatment and prophylaxis. In Brown KLB (ed): Complications of Limb Salvage. 6th International Symposium on Limb Salvage (ISOLS), Montreal, 1991, pp 511-514.

42. Cool WP, Grimer RJ, Carter SR, Sneath RS, Walker PS: Longitudinal growth following a growing endoprosthetic replacement of the distal femur in the skeletal immature. 8th International Symposium on Limb Salvage (ISOLS), Italy, 1995, p 127.

43. Safran MR, Eckardt JJ, Kabo JM, Oppenheim WL: Continued growth of the proximal part of the tibia after prosthetic reconstruction of the skeletally immature knee. J Bone Joint Surg [Am] 1992; 74:1172-1179.

44. Cool WP, Grimer RJ, Carter SR, Sneath RS, Walker PS: The outcome of extensible endoprosthetic replacements of the proximal tibia and distal femur. 8th International Symposium on Limb Salvage (ISOLS), Italy, 1995, p 126. 